\title{
Estudo de casos hospitalizados por pneumonia comunitária no período de um ano*
}

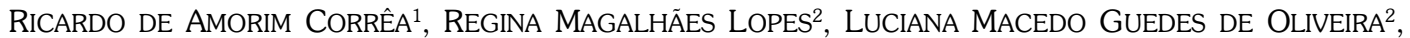 \\ Frederico Thadeu Assis Figueiredo CAmpos $^{3}$, Marco Antônio SoARes Reis ${ }^{2}$, Manoel Otávio da Costa Rocha ${ }^{4}$
}

Introdução: Apesar dos avanços obtidos nos métodos propedêuticos, cerca de $50 \%$ dos casos de pneumonia adquirida na comunidade não têm sua etiologia esclarecida, inclusive os hospitalizados. Apesar disso, a terapêutica adequada proporciona baixas taxas de mortalidade na maioria dos casos.

Objetivos: Descrever a epidemiologia, formas de apresentação, o rendimento dos testes diagnósticos, a permanência hospitalar, a morbidade e mortalidade de 42 pacientes consecutivos, internados para tratamento de PAC. Métodos: Foram incluídos pacientes com quadro clínico compatível com PAC, opacidade radiológica pulmonar recente e com dois itens entre febre, tosse produtiva e leucocitose. A solicitação de exames complementares obedeceu à necessidade de cada caso. Resultados: Dos 42 pacientes, com idade de 64,7 \pm 16,8 anos, 27 (64,3\%) masculinos, 27 (64\%) apresentavam co-morbidades. Dezessete $(40,5 \%)$ estavam em uso de antibióticos à admissão. Pneumonia grave ocorreu em oito casos (19\%); não houve diferença quanto à gravidade $(p=0,57) e$ permanência hospitalar $(p=0,25)$ entre os grupos $>$ de 60 ou $\leq$ de 60 anos. A permanência hospitalar média foi de 14,3 \pm 7,6 dias. Diagnóstico etiológico definitivo foi obtido em três casos: Legionella sp em dois, S. aureus em um caso. Em 31 (74\%), manteve-se o antibiótico inicial; em 11 $(26 \%)$ houve troca, seis $(54,5 \%)$ devido à má resposta clínica e cinco $(45,5 \%)$ devido ao resultado microbiológico. Hemoculturas foram feitas em 16 casos (38\%), positivas em apenas um (6,3\%). Nove amostras de escarro $(9 / 22,41 \%)$ foram validadas. Ocorreu um óbito $(2,4 \%)$, por pneumonia grave,

em um paciente com neoplasia. Conclusões: O diagnóstico etiológico em PAC, mesmo em internados, é obtido em uma minoria de casos, contribuindo para isso o uso concorrente de antibióticos. A terapêutica empírica adequada proporciona baixas taxas de mortalidade. Os testes diagnósticos devem ser empregados de maneira individualizada. (J Pneumol 2001;27(5):243-248)

\section{A study of community-acquired pneumonia inpatients in a period of a year}

Introduction: Besides the improvement we have had in the diagnostic methods, the causative agent in around $50 \%$ of the cases of community-acquired pneumonia (CAP) remains unknown, even in inpatients. Despite that, adequate empirical therapy results in low mortality in the majority of the cases. Goals: To describe the epidemiology, the clinical presentation, the utility of diagnostic tests, the duration of hospital stay, the morbidity and mortality rates of 42 consecutive inpatients with CAP. Methods: The inclusion criteria were the presence of a recent pulmonar infiltrate in the CXR and two items out of fever $\left(38^{\circ} \mathrm{C}\right)$, productive cough and leukocytosis $\left(>10,000 / \mathrm{mm}^{3}\right)$, in the presence of a compatible clinical syndrome. The subsidiary tests were performed as required on individual basis. Results: Forty-two patients, aged $64.7 \pm$ 16.8 years, $27(64.3 \%)$ male, were studied. Twenty-seven (64\%) had subjacent illness. Seventeen

* Trabalho realizado na Clínica de Pneumologia e Cirurgia Torácica do Hospital Madre Teresa, Belo Horizonte, MG.

1. Pneumologista; Mestrando da Disciplina de Doenças Infecciosas e Parasitárias do Curso de Pós-graduação da Faculdade de Medicina da Universidade Federal de Minas Gerais.

2. Pneumologista.

3. Coordenador da Clínica de Pneumologia e Cirurgia Torácica.
4. Professor Adjunto do Departamento de Clínica Médica da Faculdade de Medicina da UFMG; Coordenador da Disciplina de Doenças Infecciosas e Parasitárias da Faculdade de Medicina da UFMG.

Endereço para correspondência - Av. Prudente de Morais, 1.965/ 605 - 30380-000 - Belo Horizonte, MG. Tel. (31) 3297-4414; Fax (31) 3292-0942; E-mail: racorrea@uai.com.br

Recebido para publicação em 24/11/00. Aprovado, após revisão, em 1/8/01. 
$(40.5 \%)$ were on antimicrobial therapy on admission. There were eight cases (19\%) of severe pneumonia; the patients aged $>60$ or $\leq 60$ years were similar concerning the severity of presentation $(p=0.57)$ and had similar hospital stay $(p=0.25)$. The mean global hospital stay was $14.3 \pm 7.6$ days. Definite etiologic diagnosis were done in three patients: Legionella sp (2), S. aureus (1). In thirty-one cases $(73.8 \%)$ the antimicrobial therapy wasn't modified; when it happened (11 patients, 26.2\%), the main reason was the bad outcome in six cases (54.5\%); in five (45.5\%), the microbiological result prompted the change in therapy. Blood samples were drawn in 16 cases (38\%), being positive in only one (6.3\%). There were nine adequate sputum samples for analysis $(9 / 22,41 \%)$. There was only one death $(2.4 \%)$, due to severe pneumonia in a patient with a neoplastic disease. Conclusion: The etiologic diagnosis in CAP is reached in a minority of cases, even in inpatients. At least partly, this is probably due to the previous use of antimicrobial drugs. Adequate empirical therapy results in low mortality. Diagnostic tests can be performed on individual basis.

Descritores - Pneumonia. Infecções comunitárias adquiridas. Epidemiologia. Terapêutica.

Key words - Pneumonia. Community acquired infections. Epidemiology. Therapeutics.

\section{INTRODUÇÃO}

A pneumonia adquirida na comunidade (PAC) constitui importante causa de morbidade e mortalidade em todo o mundo. A despeito do avanço obtido nas técnicas de diagnóstico, estima-se que cerca de $50 \%$ dos casos ainda permaneçam sem etiologia definida ${ }^{(1-4)}$.

Em pacientes ambulatoriais, a taxa de mortalidade permanece baixa (1-5\%), mas nos que requerem hospitalização, essa taxa aumenta significativamente, particularmente se o paciente necessita admissão em unidade de terapia intensiva $^{(1-5)}$. A definição do local do tratamento constitui etapa importante da terapêutica, que repercute nos seus resultados e custos, havendo estudos recentes que sugerem critérios que auxiliam no reconhecimento de pacientes com baixa taxa de mortalidade ${ }^{(5,6)}$, passíveis de receberem tratamento em nível ambulatorial.

A escolha da terapêutica antimicrobiana é essencialmente empírica, baseando-se em dados clínicos e epidemiológicos, uma vez que a propedêutica etiológica é demorada e de baixo rendimento, não se justificando o inerente retardo na instituição da terapêutica ${ }^{(1,3,5,7)}$. Nos pacientes internados, há controvérsias quanto à investigação etiológica: para a American Thoracic Society (ATS) estaria indicada nos pacientes graves ou naqueles que não apresentam melhora com o tratamento inicial ${ }^{(1)}$; para a Infectious Diseases Society of America (IDSA), ela deveria ser feita, desde o início, nos pacientes hospitalizados ${ }^{(5,8)}$. O presente estudo apresenta uma série de 42 casos consecutivos da doença, admitidos em um hospital geral em Belo Horizonte, Minas Gerais, tendo como objetivos a descrição do seu perfil clínico e epidemiológico, das formas de apresentação da doença, do rendimento dos mé-
Siglas e abreviaturas utilizadas neste trabalho

PAC - Pneumonia adquirida na comunidade

HIV - Vírus da imunodeficiência humana

ATS - American Thoracic Society

IDSA - Infectious Diseases Society of America

todos de diagnóstico empregados, da morbidade, da mortalidade e permanência hospitalar.

\section{PACIENTES E MÉTODOS}

Estudo prospectivo, observacional, realizado na unidade de internação do Hospital Madre Teresa em Belo Horizonte, Minas Gerais, no período de maio de 1998 a maio de 1999.

\section{CRITÉRIOS DE INCLUSÃO:}

- Na presença de quadro clínico compatível com infecção respiratória aguda, o achado de um infiltrado pulmonar novo à radiografia do tórax associado a pelo menos dois dos seguintes itens:

$>$ febre $\left(\geq 38^{\circ} \mathrm{C}\right)$, tosse produtiva ou leucocitose $\left(>10.000 / \mathrm{mm}^{3}\right)$.

\section{CRITÉRIOS DE EXCLUSÃO:}

- Diagnósticos alternativos como edema pulmonar, tromboembolismo pulmonar, tuberculose pulmonar, neoplasia torácica, pneumonia nosocomial e pacientes infectados pelo HIV.

\section{DEFINIÇÕES:}

- Diagnóstico etiológico definitivo: Quando, na presença de quadro clínico compatível, houver recuperação de agente etiológico de amostra biológica não-contaminada como sangue, líquido pleural, aspirado transtraqueal ou punção transtorácica, ou demonstração direta, indireta ou através de cultura, de agente não colonizador 
(fungos não-colonizadores, micobactérias, Legionella sp) ou a presença de sorologia positiva para germe específi$\mathrm{co}$;

- Diagnóstico etiológico provável: presença de quadro clínico compatível associado à detecção, por esfregaço ou cultura, de agente provável em amostra respiratória, como escarro, aspirado brônquico, lavado broncoalveolar, escovado protegido.

- Foi considerada amostra válida de escarro, para análise ao gram e cultura, aquela que continha mais de 25 polimorfonucleares e menos de 10 células escamosas por campo de pequeno aumento.

\section{PROTOCOLO:}

Foram consideradas as seguintes variáveis: idade; sexo; presença de co-morbidades (insuficiência renal, diabetes mellitus, insuficiência cardíaca congestiva, asma, neoplasias, doença pulmonar obstrutiva crônica); fatores de risco (alteração sensorial, alcoolismo, quimioterapia, uso de corticóide e tabagismo); presença de sintomas respiratórios e sintomas gerais; apresentação radiológica; uso de antimicrobianos anterior à admissão hospitalar; sinais de gravidade (choque, acometimento radiológico multilobar, necessidade de ventilação mecânica, freqüencia respiratória maior do que 30 incursões por minuto e $\mathrm{PaO}_{2}$ menor do que $60 \mathrm{mmHg}$ ); pneumonia grave: presença de choque séptico ou necessidade do uso de ventilação mecânica;

Foram analisados também o esquema antimicrobiano utilizado, a necessidade de modificação do esquema terapêutico; exames propedêuticos individualizados que incluíram exames hematimétricos, bioquímica geral, gasometria arterial, hemoculturas, gram e cultura de escarro, análise do líquido pleural e lavado broncoalveolar broncoscópico. Foi realizada sorologia para germes atípicos em casos selecionados.

\section{ANÁlise ESTATÍSTICA}

Os dados são apresentados como média e desvio padrão, sempre que possível. Para comparação de proporções foi empregado o teste do qui-quadrado e, quando necessário (valores esperados menores do que cinco), o teste exato de Fisher. Para comparação entre médias foi empregado o teste $t$ de Student. A avaliação de risco foi feita através da razão de chances (odds ratio, OR), com intervalo de confiança de $95 \%$ de Cornfield. O nível de significância estatística foi de $5 \%(p<0,05)$ para todos os testes empregados.

\section{Resultados}

O estudo incluiu 42 pacientes, com idade de $64,7 \pm$ 16,8 anos (20-91 anos), 28 (66,7\%) dos quais com idade superior a 60 anos, sendo $27(64,3 \%)$ do sexo masculino. Sessenta e quatro por cento (27) apresentavam doenças crônicas subjacentes (Figura 1).

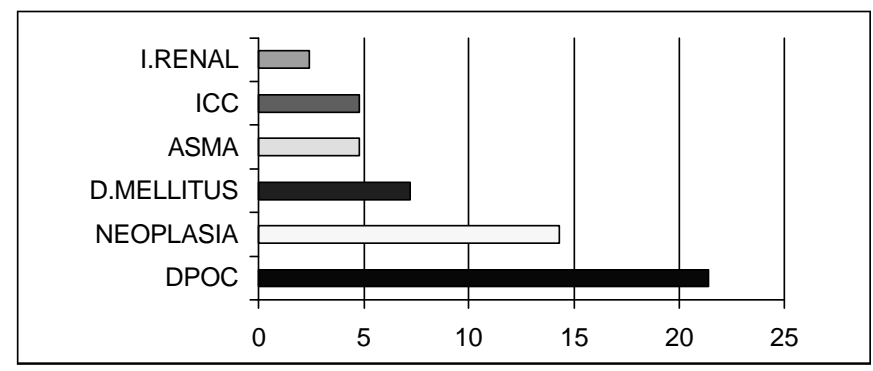

Figura 1 - Freqüência das diversas patologias subjacentes em pacientes admitidos com pneumonia comunitária

Verificou-se maior freqüência de doença subjacente em pacientes com idade superior a 60 anos $(p=0,02)$ com OR de 6,6 (IC a 95\% = 1,30-36,41). Os fatores de risco associados foram, em ordem de freqüência: tabagismo $(35,8 \%)$, alcoolismo $(7,2 \%)$, corticoterapia sistêmica $(11,9 \%)$, quimioterapia $(9,5 \%)$ e alteração sensorial $(4,8 \%)$, estando ausentes em $33,3 \%$ dos casos.

Cerca de $40 \%$ dos pacientes estavam em uso de antibiótico à admissão hospitalar. Oito casos (19\%) estavam criticamente enfermos à admissão (Tabela 1), não tendo sido observada diferença significativa da gravidade entre

TABELA 1

Características dos 42 pacientes hospitalizados no período de maio de 1998 a maio de 1999 no Hospital Madre Teresa, Belo Horizonte

\begin{tabular}{lc}
\hline \multicolumn{1}{c}{ Características } & $\mathrm{n}(\%)$ \\
\hline N úmero de pacientes & $42(100)$ \\
Sexo & \\
$\quad$ Masculino & $27(64)$ \\
Feminino & $15(36)$ \\
Idade & \\
Média \pm DP & $64,7 \pm 16,8$ anos \\
Maiores de 60 anos & $28(66,7)$ \\
60 anos ou menos & $14(33,3)$ \\
Procedência & \\
$>$ Comunidade & $38(90,5)$ \\
$>$ Ambulatórios/hospitais & $4(9,5)$ \\
U so prévio de antibióticos & $17(40,5)$ \\
M orbidade & \\
$>$ Pneumonia grave & $8(19)$ \\
Permanência hospitalar global (dias) & $14,3 \pm 7,6$ \\
$>$ M aiores de 60 anos & $13,4 \pm 6,8$ \\
$>60$ anos ou menos & $16,2 \pm 9,1 *$ \\
M ortalidade & $1(2,4)$ \\
\hline * $=0,25$ &
\end{tabular}


o grupo de pacientes acima de, ou com 60 anos ou menos $(p=0,57)$.

Os sinais de gravidade encontrados foram choque (dois casos, $5 \%$ ), acometimento radiológico multilobar em cinco (12\%), necessidade de ventilação mecânica em sete (17\%), freqüência respiratória maior do que $30 / \mathrm{min}$ em 18 (43\%), $\mathrm{PaO}_{2}$ menor do que 60mmHg em 20 (48\%), estando ausentes em 16 pacientes $(38,1 \%)$.

Tosse foi o sintoma mais freqüente (33 casos, $79 \%$ ), seguida por dispnéia em 30 pacientes (71\%), febre em $27(64,3 \%)$, dor torácica em oito (19\%). Cianose foi observada em dez pacientes (24\%).

As alterações radiológicas encontradas foram, em ordem de freqüência, consolidações únicas $(21 ; 50 \%)$, consolidações múltiplas $(10 ; 23,8 \%$ ), derrame pleural (oito; $19 \%$ ), infiltrado intersticial localizado (dois; $4,8 \%$ ) e cavitação (um; $2,4 \%$ ).

A média global de permanência hospitalar foi de 14,3 \pm 7,6 dias. Considerando-se a idade, não houve diferença estatisticamente significativa da permanência entre os grupos de pacientes com idade acima ou abaixo de 60 anos $(13,4 \pm 6,8$ dias e 16,2 \pm 9,1 dias, $p=0,25)$.

Diagnóstico etiológico definitivo foi obtido em três casos: L. pneumophila em dois, ambos com pneumonia grave na unidade de terapia intensiva (Tabela 2).

Hemoculturas foram realizadas em 16 casos (38\%), sendo positivas em apenas um $(6,3 \%)$. Foram obtidas amostras de escarro de 22 pacientes (52\%), sendo que apenas nove $(41 \%)$ foram consideradas representativas do trato respiratório inferior. A baixa freqüência de isolamento de germes não permitiu a sua comparação com a presença de co-morbidades ou com o grupo etário.

Os esquemas antibióticos utilizados estão representados na Figura 2. Em 31 casos (74\%) manteve-se o antibiótico inicial; em 11 (26\%) houve troca do mesmo, mais da metade $(54,5 \%)$ devido à resposta clínica insatisfatória e, em $45,5 \%$ das vezes, devido ao resultado microbiológi-

TABELA 2

Microorganismos identificados nos casos hospitalizados por pneumonia comunitária no Hospital Madre Teresa, Belo Horizonte, MG (maio de 1998 a maio de 1999)

\begin{tabular}{llc}
\hline \multicolumn{1}{c}{ Microorganismos } & Método de identificação & $\begin{array}{c}\mathrm{N}^{\circ} \\
\text { casos }\end{array}$ \\
\hline E. coli & Cultura de escarro & 1 \\
S. aureus oxacilino-resistente & Cultura de escarro & 1 \\
S. aureus oxacilino-sensível & Hemocultura & 1 \\
S. aureus oxacilino-sensível & Cultura de escarro & 2 \\
Legionella pneumophila & Imunofluorescência monoclonal* & 2 \\
Klebsiella pneumoniae & Cultura de escarro & 3 \\
Pseudomonas aeruginosa & Cultura de escarro & 4 \\
\hline
\end{tabular}

* Lavado brônquico $=1$; Escarro $=1$.

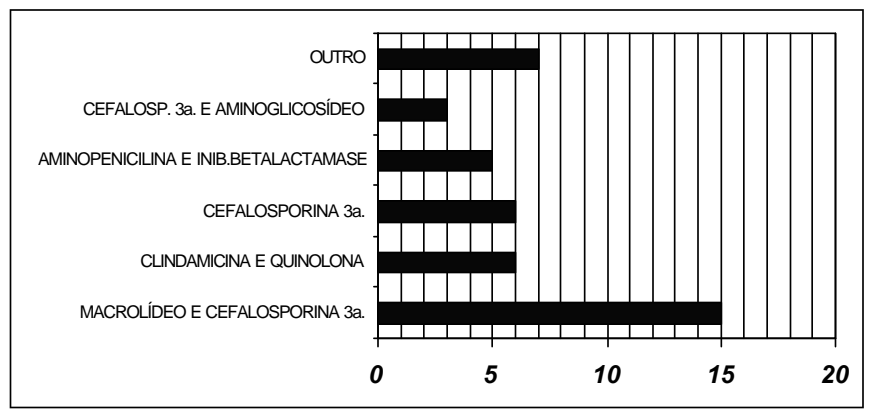

Figura 2 - Freqüência dos diversos esquemas terapêuticos utilizados nos pacientes do estudo

co. Apesar da média elevada de idade, da presença freqüente de co-morbidades e da gravidade dos casos, ocorreu apenas um óbito $(2,4 \%)$, em paciente com neoplasia e pneumonia grave por gram-negativo.

\section{DISCUSSÃO}

O presente estudo incluiu pacientes hospitalizados para tratamento de pneumonia adquirida na comunidade, com o objetivo de descrever as suas características clínicas e epidemiológicas e analisar os achados laboratoriais, radiológicos e terapêuticos utilizados, bem como a resposta obtida com o tratamento.

A faixa etária mais elevada desta amostra $(66,7 \%$ com idade superior a 60 anos) associou-se com presença freqüente de doença crônica subjacente, o que justificou parcialmente a hospitalização dos pacientes. A permanência hospitalar elevada pode também ser explicada por esses dois fatores.

Alta proporção de pacientes (14, 33,3\%) tinha 60 anos ou menos, cinco dos quais não apresentavam co-morbidades crônicas, mas com indicações clínicas de internação: dois pacientes transferidos de outro hospital, apresentando resposta insatisfatória ao tratamento (persistência de febre), ambos com derrame pleural: um necessitou toracocentese de alívio, com resolução da febre, e o outro necessitou drenagem para tratamento de empiema estafilocócico; um paciente com pneumonia grave, hipotenso e com insuficiência renal aguda à admissão; um paciente procedente de clínica asilar, deficiente mental, com acometimento radiológico multilobar e pneumotórax espontâneo hipertensivo; uma paciente em período puerperal recente, que iniciou os sintomas compatíveis com pneumonia no período pré-parto, foi admitida sem sinais de gravidade. Os demais nove pacientes apresentavam critérios de gravidade, co-morbidades e/ou fatores de risco de evolução desfavorável. Estudo retrospectivo de Fine et al. estratificou os pacientes com PAC, baseando-se na idade, procedência, presença de cinco co-mor- 
bidades e achados clínicos e laboratoriais, e sugeriu o seu uso como critério adjuvante na escolha do local de tratamento; assim, os pacientes das classes I e II poderiam ser tratados ambulatorialmente, os pacientes da classe III com uma breve internação, e os pacientes das classes IV e $\mathrm{V}$ necessitariam tratamento em regime de internação. Tal metodologia mostrou-se de melhor utilidade na identificação de pacientes de baixo risco, apresentando limitações na identificação dos de maior risco de óbito(6).

As indicações de internação verificadas no nosso estudo podem representar um viés de seleção, uma vez que este hospital é uma referência local para tratamento de doença respiratória.

Oito pacientes foram admitidos na unidade de terapia intensiva devido à necessidade do uso de ventilação mecânica e tratamento de choque séptico (um paciente). Tendo como definição de pneumonia grave a admissão na unidade de terapia intensiva, Ewig et al. ${ }^{(9)}$ analisaram prospectivamente 331 pacientes (pneumonia não-grave) com 64 pacientes (pneumonia grave) e verificaram que os critérios adotados pela ATS (1993) apresentavam alta sensibilidade (98\%), porém especificidade e valor preditivo positivo insuficientes ( $32 \%$ e $24 \%$, respectivamente) para a definição de pneumonia grave; sugeriram a adoção de dois entre três critérios menores (pressão arterial sistólica $<90 \mathrm{mmHg}$, envolvimento radiológico multilobar, $\mathrm{PaO}_{2} / \mathrm{FiO}_{2}<250$ ) ou um entre dois critérios maiores (necessidade de ventilação mecânica, presença de choque séptico), os quais apresentaram melhor performance na identificação destes casos (sensibilidade de $78 \%$, especificidade de $94 \%$, valor preditivo positivo de $75 \%$ e valor preditivo negativo de $95 \%$ ).

Quarenta por cento dos casos estavam em uso de antibióticos quando da admissão hospitalar, o que justifica o baixo rendimento obtido pela hemocultura (positiva em $1 / 16$ casos $-6,3 \%$ ) e a não-identificação dos agentes, na maioria dos casos. Um estudo, comparando pacientes hospitalizados em uso de antibiótico quando da admissão, com pacientes admitidos sem tratamento prévio, observou diferença significativa na proporção de identificação de germes, a favor do segundo grupo (27/57 casos e 149/225, respectivamente, $\mathrm{p}=0,007)^{(4)}$. Outro estudo, prospectivo, em pacientes hospitalizados, apresentou resultados semelhantes, com rendimento melhor, porém não significativo, na identificação de germes no grupo sem antibiótico à admissão $(2 / 11,11 \%$ no grupo em uso de antibiótico e 15/42, 36\% no grupo sem antibiótico, $\mathrm{p}>0,05)^{(10)}$.

Diagnóstico etiológico definitivo foi obtido em três casos: legionelose em dois (ambos com neoplasia) e pneumonia estafilocócica com empiema em um caso (3/42, $7,1 \%)$. Diversos trabalhos demonstram a alta proporção de pacientes que permanecem sem identificação etiológi- ca, a despeito da realização de extensa propedêutica ${ }^{11,3-}$ 5,10-12)

Discute-se na literatura a utilidade da realização do gram $e$ da cultura de escarro de rotina na investigação da PAC, tendo como obstáculos à sua realização, segundo a ATS, a não representatividade das vias aéreas inferiores das amostras não-validadas, a necessidade da interpretação por patologista experiente e familiarizado com a técnica, e a ausência de impacto nos desfechos do tratamento, tendo em vista a disponibilidade atual de antibióticos eficientes e seguros ${ }^{(1,7)}$. No entanto, a IDSA preconiza a sua realização, devido à possibilidade da escolha correta inicial do antibiótico e de estreitar o espectro antimicrobiano após a identificação do agente $e^{(5)}$. Em nossa casuística, a maioria das amostras não preenchia os critérios de validação, o que está de acordo com Gleckman et al., que obtiveram amostras válidas em apenas 59 de 144 pacientes admitidos com PAC $(41 \%)^{(13)}$. Em nosso estudo, em nove casos em que as amostras preenchiam os referidos critérios, a cultura de escarro isolou agentes pouco usuais, ressaltando-se o não isolamento de $S$. pneumoniae e $H$. influenzae. Isso pode ser explicado pelo uso prévio de antibióticos, pela faixa etária elevada, pela alta freqüência de co-morbidades, todos fatores que aumentam a participação etiológica de bastonetes gram-negativos ${ }^{(5)}$. A baixa prevalência dos germes "atípicos" nesta amostra pode ser explicada pela não realização de propedêutica sistemática dos mesmos, tendo sido indicada apenas em casos selecionados, notadamente quando da suspeita de legionelose em pneumonia grave.

A antibioticoterapia inicial foi mantida em $73,8 \%$ dos casos, o que comprova a adequação da abordagem empírica na maioria dos casos, já observada em outros trabalhos $^{(14,15)}$. Em estudo prospectivo, envolvendo 106 pacientes em três hospitais, Woodhead et al. relataram a necessidade de modificação do antibiótico inicial em 33 casos (31\%), sendo em 18 (54\%) destes devido às alterações clínicas, em nove (27\%) devido aos resultados microbiológicos e, em seis (19\%), por motivo não relatado ${ }^{(10)}$. De modo semelhante em nosso estudo, em 11 casos (26\%) houve necessidade da troca do esquema terapêutico inicial, sendo que em seis $(54,5 \%)$, o motivo foi a resposta insatisfatória ao mesmo e não o resultado microbiológico.

Comparando-se os dois grupos etários, tendo como divisor a idade de 60 anos, não observamos diferença significativa quanto à gravidade da pneumonia $(\mathrm{p}=0,57)$. Também não houve diferença estatística entre os grupos etários quanto à permanência hospitalar $(\mathrm{p}=0,25)$, o mesmo ocorrendo quando a comparamos quanto à presença ou ausência de co-morbidades $(p=0,52)$.

Os achados radiológicos encontrados não puderam ser correlacionados com a etiologia, devido ao pequeno número de diagnósticos definitivos obtidos. Em diversas sé- 
ries publicadas, inclusive em recente trabalho em nosso meio, que estudou pacientes ambulatoriais, não foram encontrados achados radiológicos definitivos quanto à probabilidade do diagnóstico dos diversos agentes etiológicos da PAC ${ }^{(16)}$.

O esquema antibiótico mais utilizado foi a combinação de macrolídeos com uma cefalosporina de terceira geração, uma das opções sugeridas em diretrizes de tratamento em publicações de consensos para pacientes hospitaliza$\operatorname{dos}^{(1,5)}$. Os demais esquemas obedeceram ao esquema inicial já em uso pelos pacientes. Quinolonas com atividade antipneumocócica não foram utilizadas por não constarem da padronização de medicamentos em vigor no hospital à época do estudo. Em três casos utilizou-se aminoglicosídeo associado a cefalosporina de terceira geração, devido à sua ação sinergística contra o bastonete gram-negativo identificado.

Apesar da gravidade da amostra estudada, observamos baixa taxa de mortalidade $(2,4 \%)$, o que comprova a eficiência do tratamento empregado.

\section{CONCLUSÕES}

Na amostra estudada, o uso de antibióticos previamente à admissão hospitalar parece ter contribuído para o não reconhecimento dos agentes. Nos casos em que se obteve amostra adequada de secreção respiratória para cultura, o resultado microbiológico motivou a troca de antibiótico em apenas $45,5 \%$ dos 11 casos em que esta ocorreu. O tratamento instituído de maneira empírica mostrou-se altamente eficaz, demonstrado pela baixa taxa de mortalidade observada. Os resultados obtidos não podem ser generalizados, devido às particularidades da amostra estudada. A abordagem diagnóstica e a terapêutica na PAC devem ser individualizadas, devendo ser perseguido o diagnóstico etiológico nos pacientes internados e naqueles com pneumonia grave desde o início.

\section{REFERÊNCIAS}

1. American Thoracic Society. Guidelines for the initial management of adults with community-acquired pneumonia: diagnosis, assessment of severity, and initial antimicrobial therapy. Am Rev Respir Dis 1993; $148: 1418-1426$
2. British Thoracic Society. Guidelines for the management of community-acquired pneumonia in adults admitted to hospital. Br J Hosp Med 1993;49:346-350.

3. Fine MJ, Smith MA, Carson CA, Mutha SS, Sankey SS, Weissfeld LA, et al. Prognosis and outcomes of patients with community-acquired pneumonia. JAMA 1996;275:134-141.

4. Fang GD, Fine M, Orloff J, Arisumi D, Yu VL, Kapoor W, et al. New and emerging etiologies for community-acquired pneumonia with implications for therapy: a prospective multicenter study of 359 cases. Medicine (Baltimore) 1990;69:307-316.

5. Bartlett JG, Dowell SF, Mandell LA, File TM, Musher DM, Fine MJ. Practice guidelines for the management of community-acquired pneumonia in adults. Clin Infect Dis 2000;31:347-382.

6. Fine MJ, Auble TE, Yealy DM, Hanusa BH, Weissfeld LA, Singer DE, et al. A prediction rule to identify low-risk patients with communityacquired pneumonia. N Engl J Med 1997;336:243-250.

7. Niederman MS. Empirical therapy of community-acquired pneumonia. Semin Respir Infect 1994;9:192-198.

8. Bartlett JG, Breiman RF, Mandell LA, File Jr TM. Community-acquired pneumonia in adults: guidelines for management. Clin Infect Dis 1998; 26:811-838

9. Ewig S, Ruiz M, Mensa J, Marcos MA, Martinez JA, Arancibia F, et al. Severe community-acquired pneumonia. Assessment of severity criteria. Am J Respir Crit Care Med 1998;158:1102-1108

10. Woodhead MA, Arrowsmith J, Chamberlain-Webber R, Wooding S, Williams I. The value of routine microbial investigation in communityacquired pneumonia. Respir Med 1991;85:313-317.

11. Marrie TJ, Peeling RW, Fine MJ, Singer DE, Coley CM, Kapoor WN. Ambulatory patients with community-acquired pneumonia: the frequency of atypical agents and clinical course. Am J Med 1996;101:508515 .

12. Fine MJ, Stone RA, Singer DE, Coley CM, Marrie TJ, Lave JR, et al. Processes and outcomes of care for patients with community-acquired pneumonia. Arch Intern Med 1999;159:970-980.

13. Gleckman R, DeVita J, Hibert D, Pelletier C, Martin R. Sputum gram stain assessment in community-acquired bacteremic pneumonia. J Clin Microbiol 1988;26:846-849.

14. Meehan TP, Fine MJ, Krumholz HM, Scinto JD, Galusha DH, Mockalis JT, et al. Quality of care, process and outcomes in elderly patients with pneumonia. JAMA 1997;278:2080-2084

15. Gleason PP, Meehan TP, Fine MJ, et al. Associations between initial antimicrobial agents regimens and medical outcomes for elderly patients with pneumonia. Arch Intern Med 1999;159:2562-2572.

16. Rocha RT, Vital AC, Silva COS, Pereira CAC, Nakatani J. Pneumonia adquirida na comunidade em pacientes tratados ambulatorialmente: aspectos epidemiológicos, clínicos e radiológicos das pneumonias atípicas e não atípicas. J Pneumol 2000;26:5-14. 